\title{
La difusión de los presupuestos románticos a través de dos revistas artísticas decimonónicas: El Artista (1835-1836) y The Germ (1850)
}

\author{
María Victoria Álvarez Rodríguez \\ Universidad de Salamanca \\ mvalvarez@usal.es
}

RESUMEN: El surgimiento de las revistas artísticas fue una de las aportaciones más originales de la Europa decimonónica a la historiografía artística contemporánea. Estrechamente relacionadas con la defensa de los ideales románticos en algunos casos, orientadas hacia el gran público en otros, esas revistas contribuyeron decisivamente a que la sociedad burguesa del siglo XIX entrara en contacto con las Bellas Artes que hasta entonces habían permanecido encerradas en círculos académicos. En el presente análisis nos proponemos comparar críticamente dos proyectos periodísticos que nacieron en el seno de círculos artísticos adscritos al Romanticismo, la revista española El Artista (18351836) y la inglesa The Germ (1850), evaluando hasta qué punto contribuyeron a difundir los ideales de dicho movimiento, qué estrategias se siguieron en cada caso y qué grado de compromiso se aprecia en sus equipos directivos, estableciendo similitudes y diferencias en función del estadio y evolución del movimiento romántico en ambos países.

PALABRAS CLAVE: Prensa artística, El Artista, The Germ, Siglo XIX, Romanticismo, España, Inglaterra.

\section{The Spread of Romantic Postulates through the Nineteenth-Century Art Journals. El Artista (1835-1836) and The Germ (1850)}

ABSTRACT: The birth of artistic magazines was one of the most original contributions of nineteenth-century Europe to the contemporary art historiography. Closely related to the defense of the romantic ideals in some cases, oriented towards the general public in others, these magazines contributed decisively towards the bourgeois society of the nineteenth century's contact with the Fine Arts which had hitherto remained locked in academic circles. In this analysis we propose to compare significantly two journalistic projects born within artistic circles attached to Romanticism, the Spanish magazine El Artista (1835-1836) and the English The Germ (1850), evaluating to what extent they helped to spread the ideals of this movement, what strategies were followed in each case and what degree of commitment showed in their management teams, establishing similarities and differences depending on the state and evolution of the Romantic movement in both countries.

KEYWORDS: Art Press, El Artista, The Germ, 19 ${ }^{\text {th }}$ Century, Romanticism, Spain, England.

Recibido: 14 abril de 2016 / Aceptado: 24 de junio de 2016.

\section{Introducción}

La historiografía artística asistió durante el siglo XIX, a nivel internacional, al fenómeno de la proliferación de numerosas publicaciones periódicas en las cuales las Bellas Artes gozaron de un papel preeminente. Consecuencia en gran medida de una progresiva democratización de la cultura, estos rotativos se convirtieron en objeto de interés del gran público, en el caso de las publicaciones más multidisciplinares y divulgativas, así como de los colectivos de jóvenes artistas en el de las revistas más

ÁLVAREZ RODRÍGUEZ, María Victoria: «La difusión de los presupuestos románticos a través de dos revistas artísticas decimonónicas: El Artista (1835-1836) y The Germ (1850)», Boletín de Arte, n. ${ }^{\circ}$ 37, Departamento de Historia del Arte, Universidad de Málaga, 2016, pp. 61-70, ISSN: 0211-8483. 
comprometidas con la difusión de sus ideales, concretamente los del Romanticismo. Esto último fue lo que sucedió con las dos revistas objeto de nuestra atención, la española El Artista (publicada entre 1835 y 1836) y la inglesa The Germ (publicada en 1850), dos de los ejemplos más paradigmáticos de esas revistas de impronta romántica que gozaron de una vida breve pero que se convirtieron aun así en punto de encuentro para un colectivo de creadores que estaban comenzando por entonces sus carreras. No obstante, conviene partir de la premisa, como comprobaremos a continuación, de que la situación que estaba atravesando la prensa no era la misma en ambos países. Lo mismo sucedió con la evolución del Romanticismo español y el inglés, condicionando decisivamente en ambos casos la idiosincrasia de estos proyectos.

En el caso español, la conquista de la libertad de prensa fue más tardía y paulatina que en el inglés, debido principalmente al aislamiento cultural en que estuvo sumido el país durante los diecinueve años en los que Fernando VII (1784-1833) ocupó el trono. Convencido de que cualquier idea procedente de más allá de los Pirineos podía suponer una amenaza al sistema político español, el monarca se aseguró de mantener a raya las iniciativas que se estaban desarrollando en el extranjero, entre ellas el origen y desarrollo de la prensa periódica en Francia. No obstante, la presencia de numerosos exiliados en la nación vecina, los viajes realizados por los intelectuales y, finalmente, la implantación de la doctrina liberal con la regencia de su viuda María Cristina de Borbón-Dos Sicilias (1806-1878), comenzaron a cambiar esta situación. Dichos cambios alcanzaron su auge durante el reinado de su hija Isabel II (1806-1878), cuando se hizo posible la aprobación de una serie de medidas como la Ley de Prensa de 1837 y la Ley Nocedal de 1857. Gracias a esas medidas comenzó a publicarse un abanico cada vez mayor de revistas en nuestro país, incorporando en muchos casos ideas progresistas en materia artística (Blanco, 1988: 43). A esto se sumó el regreso de los exiliados de mentalidad liberal de los que hemos hablado antes, muchos de los cuales habían asistido personalmente al auge del Romanticismo en Francia y estaban al tanto de lo que perseguían los artistas de dicho movimiento. Es importante tener en cuenta todas estas circunstancias porque permiten comprender por qué publicaciones como El Artista, de la que hablaremos a continuación, se mostraron más combativas en sus inicios que otras foráneas que les sirvieron de inspiración. Fue ese aisla- miento de la época fernandina con respecto al extranjero lo que retrasó la asimilación de los presupuestos románticos, y sus equipos directivos consideraron desde un principio que era su deber romper una lanza por ellos.

Por el contrario, en Inglaterra el principal problema al que debían enfrentarse los directores de las revistas no era la censura, pues en los siglos XVII y XVIII ya existía una considerable libertad de prensa con el amplísimo catálogo de títulos que implicaba esto, sino los elevados impuestos que se veían obligados a pagar. Fue una consecuencia de la Ley del Timbre aprobada en 1712 durante el reinado de Ana de Gran Bretaña (1665-1714), última soberana de la Casa Estuardo. Esta medida incrementó notablemente el precio de venta de las revistas, lo que suponía un problema considerable en el caso de las que estaban dirigidas al gran público y habían nacido por tanto con voluntad de resultar baratas. No es de extrañar que se produjera, en consecuencia, una proliferación de rotativos impresos en papel sin timbrar que pretendían escapar a aquella normativa, por lo general de tono muy revolucionario. Con el paso del tiempo, no obstante, nos encontramos con que los impuestos fueron reducidos en 1836 y finalmente, durante el reinado de Victoria del Reino Unido (1819-1901), abolidos por completo en 1855. Para entonces la prensa periódica ya había alcanzado un desarrollo incuestionable, publicándose desde la década de 1830 revistas dedicadas a los temas más variados: ciencias, religión, literatura y, por supuesto, a las Bellas Artes (Palmegiano, 2013: 3-4).

Estas fueron las circunstancias en las que vieron la luz El Artista en España y The Germ en Inglaterra. Como comprobaremos al analizar en detalle las características de cada revista, existe una vinculación insoslayable con el momento histórico concreto en que ambas salieron a la calle y con la situación cultural y artística en que se hallaban sus países. De ahí que encontremos muchos rasgos comunes en ellas, sobre todo la defensa de los ideales románticos, pero también diferencias derivadas del posicionamiento particular de sus equipos directivos y de las coyunturas específicas española y británica.

\section{El Artista (1835-1836)}

Considerada la revista más emblemática del Romanticismo español, esta iniciativa periodística surgió de la mano del 
pintor Federico de Madrazo (1815-1894) y el escritor Eugenio de Ochoa (1815-1872) [1]. Además de mantener fuertes lazos de amistad entre sí, en el año en que vio la luz El Artista se convirtieron en familiares, dado que Ochoa se casó con Carlota, la hermana de Federico, pasando a formar parte de este modo del poderoso clan familiar de los Madrazo. Ambos tenían en común su afán por difundir en España los presupuestos del movimiento que habían conocido en París. Efectivamente, en los años anteriores Federico de Madrazo había disfrutado de una estancia formativa en dicha ciudad para estudiar pintura antes de marcharse a Roma para hacer lo propio (Lafuente, 1987: 469), mientras que Eugenio de Ochoa había estudiado en la École des Beaux-Arts pese a que tuvo que abandonar sus estudios por culpa de una enfermedad (Torres, 2012: 241-243). Los dos contaron a la hora de fundar la revista con el apoyo económico de José de Negrete y Cepeda, conde de Campo-Alange (1812-1836), que compartía con ellos el deseo de dar forma a una suerte de tribuna artística, literaria y cultural desde la cual pudieran darse a conocer en España las inquietudes románticas de una nueva generación, que hasta entonces no había poseído ningún órgano de representación en el que vieran plasmados sus ideales (Ayala, 2002: 35).

Considerando esa relación que Madrazo y Ochoa poseían con el ambiente cultural parisino en el que habían estado inmersos, no es de extrañar que a la hora de crear El Artista tomaran como modelo inmediato de inspiración su homónima francesa, titulada L'Artiste, journal de la littérature et des beaux-arts, fundada en 1831 por el pintor Achille Ricourt (1797-1879) (Alonso, 2014: 102). Esta revista se estuvo publicando durante mucho más tiempo que la española, concretamente hasta 1904, pero inicialmente sus características resultan muy similares, tanto que suele afirmarse que en un primer momento Madrazo y Ochoa no hicieron sino reproducir escrupulosamente el modelo de L'Artiste con alguna excepción en viñetas y pequeños detalles de formato (Calvo y González, 1981: XVII y Simón, 1947: 261-267). No obstante, otros estudiosos como Vicente Llorens sostienen que pueden apreciarse diferencias notables en cuanto al contenido, ya que El Artista español concedía más importancia a la poesía además de tener un carácter más combativo. Esto se debió probablemente a que L'Artiste francés salió a la luz cuando en Francia ya habían sido asimilados los nuevos gustos artísticos, mucho antes que en España (1989: 259).

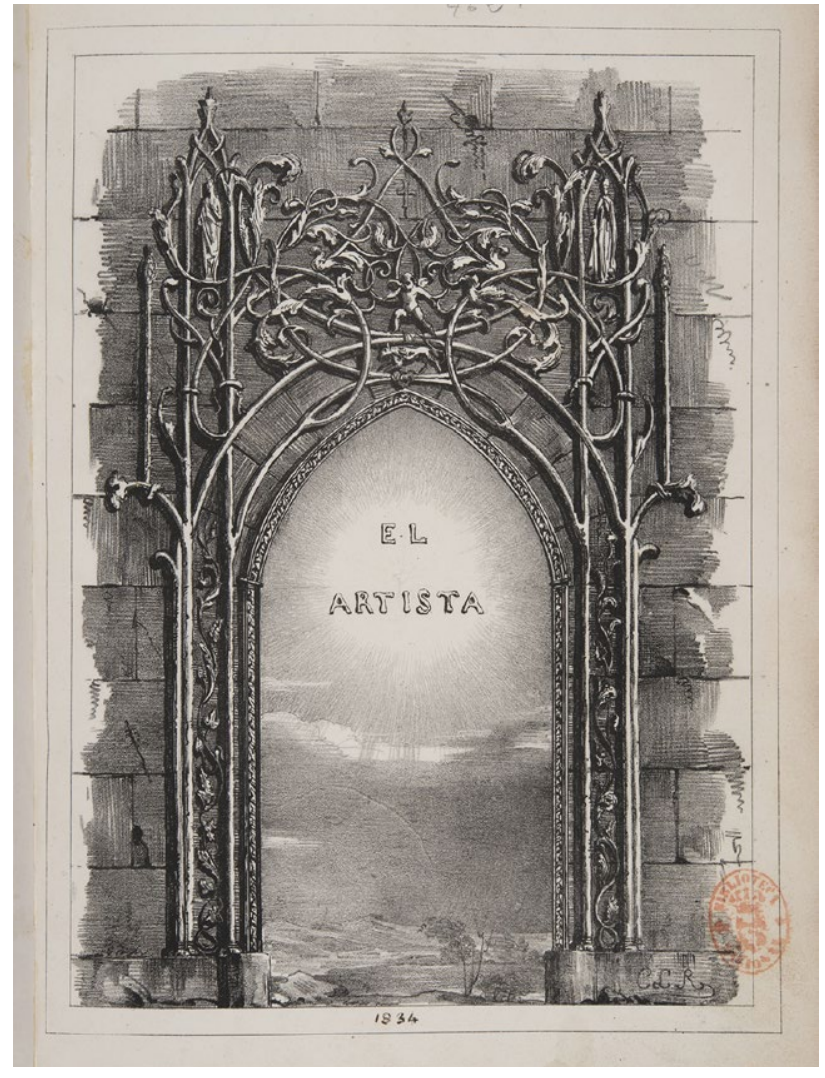

1. Frontispicio del primer tomo de la revista El Artista, 1835

Centrándonos en la publicación propiamente dicha, cuando se puso a la venta lo hizo con el título de El Artista: periódico semanal (de artes, literatura, historia, etc.). Vio por primera vez la luz el 5 de enero de 1835, continuando con una periodicidad semanal durante sesenta y cinco entregas hasta tocar a su fin el 4 de abril de 1836. Cada una de esas entregas salía a la calle los domingos y podía adquirirse por diez reales, costando la lámina suelta cinco reales. Asimismo, Ochoa y Madrazo ofrecieron la posibilidad a sus lectores de suscribirse en Madrid a treinta reales por un mes, setenta y ocho por tres meses, ciento treinta y ocho por seis meses y doscientos cuarenta por año. En el caso de la suscripción en provincias, los costes ascendían a treinta y cuatro reales por un mes, noventa por tres meses, ciento sesenta y dos por seis y doscientos ochenta y ocho por año (Hartzenbusch, 1894: 47). A diferencia de lo que encontraremos en revistas artísticas españolas de las siguientes décadas, como El Arte 
en España de Gregorio Cruzada Villaamil (1832-1884) publicada entre 1862 y 1870, El Artista no podía ser adquirida aún desde el extranjero, siendo habitual en algunas publicaciones hacerlo desde París y Nueva York.

En lo concerniente a sus características técnicas, El Artista se mantuvo apegada a la tradición de la época al poseer un formato en $4^{\circ}$ de 290 milímetros de alto por 210 milímetros de ancho, con texto a dos columnas y más de cien ilustraciones. La notable importancia que se le dio a esto último resulta lógica, considerando que se trataba, como hemos dicho, de una publicación dirigida específicamente a los círculos artísticos de la capital, y por tanto era necesario que fuera atractiva no solo en cuanto a su contenido sino también en cuanto a su continente. Por ello se decidió imprimir la revista en los prestigiosos talleres tipográficos de J. Sancha, con las estampas, grabados y cabeceros tipográficos incluidos en las páginas, mientras que las dos estampas litografiadas de mayores dimensiones que se entregaban con cada ejemplar se imprimieron en el Real Establecimiento Tipográfico que había dirigido José de Madrazo y del que entre 1835 y 1836 , los mismos años que pasó al frente de El Artista, se encargó su hijo Federico (Rodríguez, 2011: 453-455).

Merece la pena tener en cuenta asimismo la marcada diferencia que encontramos entre el soporte gráfico de El Artista y el de otras publicaciones periódicas hispanas coetáneas que, a diferencia de la que nos ocupa, se encontraban dirigidas al gran público. Lo más frecuente en esos casos era que se incluyeran estampas realizadas mediante la técnica xilográfica, debido principalmente a dos motivos: la mayor rapidez de ejecución que se obtenía trabajando la madera y el menor coste que esto suponía para las revistas. De nuevo nos encontramos ante un recurso tomado del extranjero, concretamente de Francia; se sabe, de hecho, que el fundador de Semanario Pintoresco Español, el escritor Ramón de Mesonero Romanos (1803-1882), llegó a enviar a los artistas encargados de realizar las estampas de su revista al país vecino para que aprendieran la técnica en la École des Beaux-Arts de París y convirtieran así al Semanario en un pionero en este sentido (Riego, 2001: 130-133).

En el caso de El Artista, lo que primaba no era la economía sino el lujo, de ahí que se prestara una atención mucho mayor a la calidad de las estampas y estas se realizaran mediante la técnica litográfica del grabado sobre piedra. De ello se encargaron, además del propio Federico de
Madrazo, otros artistas como Francisco Aranda y Delgado (1807-1858), Carlos Luis de Ribera y Fieve (1815-1891) y Pharamond Blanchard (1805-1873). Realizadas con aguatinta, lápiz, pluma, pincel y rascador, e impresas sobre papel de China pegado con engrudo a un refinado papel blanco, estas estampas llegaron a ser tan apreciadas por parte de los suscriptores que muchos se desentendieron de la publicación para dedicarse a coleccionar los grabados. Como sostiene Gallego Gallego, en ellos se empieza a vislumbrar por primera vez el auténtico carácter romántico que más adelante se extenderá por la estampa española, empleando recursos que acabarían siendo propios de este movimiento como la tendencia a exagerar las proporciones de los edificios, los efectistas juegos de luces y sombras, la inclusión de figuras costumbristas... (1990: 349).

Sin embargo, este preciosismo acabó siendo a la larga una de las causas de que la aventura periodística de El Artista no alcanzara el año y medio de duración. Los elevados costes que suponía, agravados por el exiguo número de suscriptores y por la temprana muerte del conde de Campo-Alange en 1836 en la Primera Guerra Carlista, dieron al traste con el proyecto (Seoane, 2013: 11-43). Es uno de los ejemplos más representativos de la diferencia que hubo en la España isabelina entre las revistas orientadas a un público específico, por lo general de elevada cualificación, y las que pretendían alcanzar al conjunto de la sociedad, que al contar con un número de suscriptores mucho mayor perduraron durante más tiempo. Los casos más paradigmáticos en este sentido serían Semanario Pintoresco Español, que se estuvo publicando de 1836 a 1857, y Museo de las Familias, de 1843 a 1870.

A pesar de todo, el ocaso de El Artista en 1836 no supuso un punto y final en los proyectos periodísticos de Ochoa y Madrazo. Once años más tarde, en 1847, ambos se pusieron de nuevo al frente de una nueva publicación titulada El Renacimiento que no duró más que tres meses, desde marzo hasta julio. Compartía con El Artista tanto el preciosismo en cuanto a su formato como su orientación decididamente artística, pese a que el tono de los artículos hubiera cambiado. El Romanticismo exaltado que Ochoa y Madrazo se habían comprometido a defender desde su primera tribuna (cosa que, como veremos más adelante, no fue del todo cierta) se había convertido en la segunda en un carácter mucho más reposado, desentendiéndose de los 
excesos sentimentales propios de la juventud. Esta evolución resulta lógica si tenemos en cuenta hasta qué punto había mejorado la situación de ambos directores; por entonces Madrazo era uno de los principales pinceles del país, llegando a ser nombrado pintor de cámara de la Casa Real y director del Museo del Prado como su padre, mientras que Ochoa ostentaba los cargos de consejero de Estado y académico de la lengua, pruebas de lo firmemente instalados que se encontraban ambos en el sistema cultural de nuestro país (Rodríguez, 2004: 77).

\section{The Germ (1850)}

En el caso de esta revista inglesa, surgió asimismo en un círculo de artistas unidos tanto por estrechos lazos de amistad como por compartir unas inquietudes creativas muy concretas. The Germ fue creada en el contexto artístico del Londres victoriano por algunos de los principales nombres de la Hermandad Prerrafaelita, fundada en 1848 por los pintores Dante Gabriel Rossetti (1828-1882), John Everett Millais (1829-1896) y William Holman Hunt (1827-1910) [2]. Como su propio nombre indica, la convicción de que el arte anterior a Raffaele Sanzio (1483-1520) había poseído una sinceridad y una pureza que a partir de entonces se habían desvirtuado llevó a estos jóvenes a plantear un regreso a los ideales artísticos tardomedievales y quattrocentistas, un posicionamiento que no fue comprendido por parte de la sociedad victoriana, para la cual esta corriente pecaba de una excesiva soberbia (Bullen, 2009: 68).

El interés de los prerrafaelitas por crear una suerte de sociedad secreta, firmando sus obras con las iniciales P.R.B. (de Pre-Raphaelite Brotherhood) y reuniéndose de manera periódica con otros artistas que compartían sus mismas inquietudes, se tradujo en 1850 en el deseo de dar forma a un órgano de representación de dicho ideario. Es similar a lo que habían llevado a cabo Madrazo y Ochoa en el caso de El Artista español a modo de palestra del ideario romántico pese a que, como analizaremos más adelante, la manera de configurar estas tribunas no fuera la misma. En el caso The Germ, los promotores fueron Hunt, Rossetti y el hermano de este último, el escritor y crítico William Michael Rossetti (18291919), que se convertiría en director de la publicación (Riede, 2007: 308). No obstante, el resto del círculo prerrafaelita,

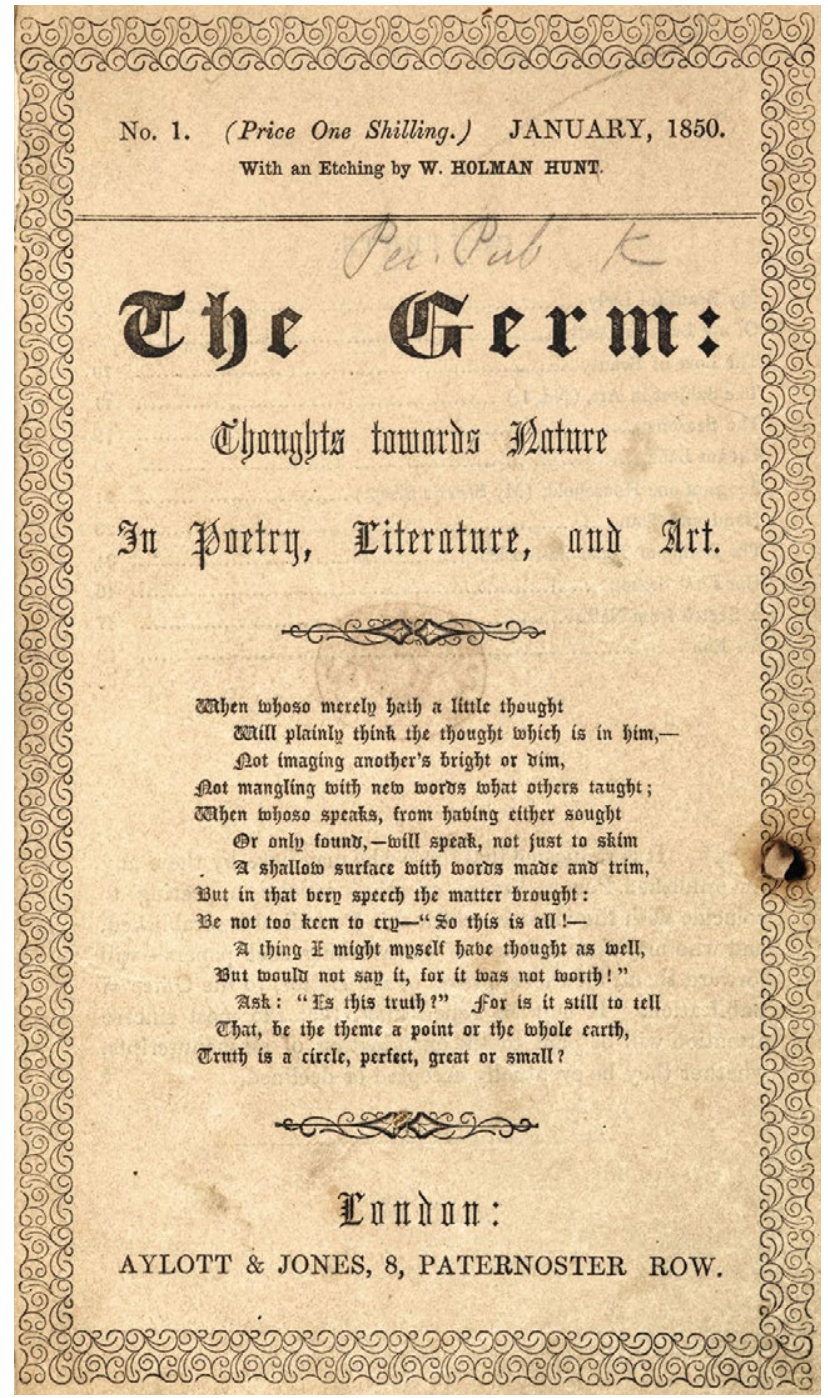

2. Frontispicio del primer tomo de la revista The Germ, 1850

en el que podemos encontrar nombres de artistas como los del mentado Millais, James Collinson (1825-1881), Thomas Woolner (1825-1892), Ford Madox Brown (1821-1893) y Walter Deverell (1827-1854), se implicó también en esta empresa, debatiendo acerca de cuáles deberían ser sus características, contribuyendo con sus propios textos y realizando grabados para acompañarla (Stauffer, 2012: 76). Como se observa, todos ellos estaban entre los veinte y los treinta años en el momento en que The Germ vio la luz, lo que se tradujo en un enorme entusiasmo por el proyecto pero, al mismo tiempo, en una falta de experiencia en cuanto a su gestión. 
En lo concerniente al título con el que la revista salió a la calle, podemos apreciar un cambio que demuestra hasta qué punto los Rossetti y su círculo eran conscientes de la acuciante necesidad de alcanzar un mayor número de lectores a fin de evitar que la publicación dejara de existir debido a problemas en su financiación. En un principio se tituló The Germ en alusión a la creencia, típicamente prerrafaelita en su relación con la naturaleza y su preocupación por difundir su ideario, de que el rotativo tenía que ser la semilla de la que surgieran nuevos artistas y creaciones afines a este. Al mismo tiempo, el subtítulo Thoughts towards Nature in Poetry, Literature and Art coincide de nuevo con sus inquietudes, pues no en vano los adalides del movimiento, sobre todo Dante Gabriel Rossetti, destacaron por dedicarse tanto a la poesía como a la pintura en un deseo de dar forma a la obra de arte total (Tobin, 2005: 3). Sin embargo, este primer título, como hemos dicho, acabó cambiando en los dos últimos números de la publicación, pasando a denominarse Art and Poetry, Being Thoughts towards Nature, Conducted Principally by Artists para especificar de esta manera, con mayor claridad, a qué público se hallaba dirigida (Holmes, 2015: 693).

La periodicidad de The Germ no era semanal sino mensual, algo lógico si tenemos en cuenta la lucha continua que estaban librando por entonces los prerrafaelitas a fin de darse a conocer en la sociedad victoriana. Su extensión, no obstante, era de cuarenta y ocho páginas, nada despreciable en comparación con otras revistas de la época. Cada ejemplar costaba un chelín y salía de la imprenta Aylott \& Jones, situada en el número 8 de Paternoster Row y dirigida por la firma Tupper \& Sons especializada en la impresión litográfica. Uno de los miembros de la familia, George Isaac Frederick Tupper (1821-1911), mantenía estrechos lazos de amistad con el círculo prerrafaelita y se encargó de correr con la mayoría de los gastos derivados de la edición, sobre todo cuando fue evidente que la empresa estaba siendo un fracaso y las aportaciones particulares de los colaboradores no bastaban para financiarla (Read, Barnes y Christian, 1991: 66).

Aunque más adelante nos detendremos en esta cuestión, conviene recordar ahora que de los cuatro ejemplares que salieron a la venta (el primero lo hizo el 1 de enero de 1850, el segundo el 31 de enero, el tercero el 31 de marzo y el cuarto el 30 de abril) se imprimieron muchas más copias de las que acabaron siendo adquiridas. En un principio, haciendo gala de un excesivo optimismo, se pusieron en circu- lación setecientos ejemplares de los que solo se vendieron setenta, de ahí que en el caso del segundo número solo se imprimieran quinientos de los que se colocaron en el mercado cincuenta. Aunque se desconocen las cifras alcanzadas por los dos últimos números, la resignación con la que fue abandonado este proyecto indica que la situación no mejoró (Rossetti y Fredeman, 1975: 39).

La publicación poseía un formato en $8^{\circ}$ de 224 milímetros de alto por 145 milímetros de ancho con texto a una sola columna, acompañado por los adornos que eran habituales en estos rotativos: pequeñas barras de separación, letras capitales de mayor tamaño en los títulos de los textos, etc. Un rasgo estilístico que de nuevo vuelve a ser puramente prerrafaelita es la recurrencia de elementos de corte medieval en dichos adornos, como la tipografía gótica de los títulos y la orla que enmarca la composición de la primera página de cada ejemplar que, a diferencia del resto, se imprimía en papel de color crema. En cuanto a los grabados que acompañaban a The Germ, encontramos que poseían una enorme importancia para el equipo directivo, algo totalmente lógico si tenemos en cuenta que casi todos sus miembros eran pintores y por tanto se encargaron personalmente de crearlos. Impresos mediante la técnica litográfica sobre piedra en la que se había especializado la firma de los Tupper, fueron realizados por William Holman Hunt, James Collinson, Ford Madox Brown y Walter Deverell. Fueron estampados en papel de India cincuenta ejemplares del primer número y más tarde, debido a esas dificultades financieras señaladas, aparecieron ya en papel normal (Suriano, 2005: 321).

El ocaso de The Germ fue decididamente temprano, aunque no cogió por sorpresa a ninguno de sus impulsores. Conviene recordar que, cuando comenzó a publicarse, la Hermandad Prerrafaelita todavía no había cumplido los dos años de vida. Por entonces sus integrantes se estaban enfrentando a las malas críticas granjeadas por sus primeras obras antes de que el teórico y crítico de arte John Ruskin (1819-1900) comenzara a mostrarles públicamente su apoyo, lo que a la postre les permitió darse a conocer y, con el paso de los años, alcanzar una proyección cada vez mayor (Cook y Wedderburn, 1904: 319-335). Esta fama postrera fue la razón de que, si bien The Germ fue un rotundo fracaso en el momento de su publicación, se reeditara medio siglo más tarde con un gran éxito. Volvió a ver la luz en 1898, más tarde en 1901 con un estudio introductorio de William 
Michael Rossetti y recientemente, en 1992, en una edición del Ashmolean Museum de Oxford (Rose, 1992). Asimismo, a finales de la centuria decimonónica vieron la luz las primeras investigaciones dedicadas a la revista, como el artículo «A Pre-Raphaelite Magazine» publicado por el escritor y ensayista James Ashcroft Noble (1844-1896) en Fraser's Magazine $(1882,568-580)$ y «The Life and Death of The Germ» publicado por el poeta y crítico Ernest Radford (1857-1919) en Idler (1898: 227-233) con motivo del cincuenta aniversario de la fundación de la Hermandad.

\section{El posicionamiento de El Artista y The Germ en la defensa de lo romántico}

Una vez explicadas las circunstancias que rodearon a ambas publicaciones en el momento de su irrupción en el panorama periodístico, nos ocuparemos de los puntos en común y las diferencias apreciables entre ellas y en el contexto en el que vieron la luz.

La principal similitud salta a la vista: tanto El Artista como The Germ pueden ser consideradas en sus respectivos países el paradigma de revista artística decimonónica en base a tres aspectos: conceder un gran protagonismo en sus páginas a las Bellas Artes, haber nacido en un círculo de artistas (tanto literatos como pintores) con inquietudes e intereses comunes y estar orientadas a un público compuesto preferentemente por artistas. Esa doble naturaleza literario-artística es una de las señas de identidad de ambas, y de hecho queda reflejada en los títulos completos, con ciertos matices que permiten distinguir su propia individualidad. Si bien en El Artista: periódico semanal (de artes, literatura, historia, etc) se hacía hincapié en esos dos temas, sumándole el componente histórico derivado del gran interés que poseía su equipo directivo por el momento que atravesaba España, en The Germ: Thoughts towards Nature in Art and Literature encontramos una mayor apuesta por la relación de la Naturaleza con el arte y la literatura. Como explicamos antes, esa fascinación resulta muy prerrafaelita y es lo que determinó el nombre de la publicación que aspiraba a ser el «germen», la «simiente» o la «semilla» de la que brotaran futuras manifestaciones artísticas afines a su ideario.

Asimismo, resulta interesante analizar las semejanzas y las diferencias que ambas revistas poseían en cuanto a su posicionamiento en el debate clasicismo-romanticismo, convertido en una constante en los círculos culturales del siglo XIX a nivel internacional. Tanto El Artista como The Germ eran conscientes de su importancia como órgano de difusión de las ideas románticas, lo cual quedó plasmado desde el primer momento en su estética, en el caso de la primera en el frontispicio en el que aparecía representado un arco ojival y, en el caso de la segunda, en la orla de resabios también medievales. No obstante, el análisis de los artículos sobre Bellas Artes publicados en ambas permite comprender que ese posicionamiento romántico fue más consecuente en el caso de The Germ, cuyos colaboradores no escondieron nunca su admiración por el arte medieval. En El Artista, por el contrario, el Romanticismo más exaltado no se encontraba presente en los textos dedicados a las Bellas Artes, sino solamente en los de temática literaria.

La razón de ser de esto es sencilla: en las fechas en las que El Artista salió a la calle el Romanticismo aún no había arraigado en España en cuanto a lo artístico, como hemos dicho, mientras que la literatura comenzaba a avanzar por la senda defendida por Ochoa y Madrazo. Este contraste se aprecia claramente en la producción de ambos directores: en esas mismas fechas de 1835 y 1836 Ochoa estaba escribiendo relatos tan románticos como Stephen o Zenobia, publicados en El Artista y repletos de elementos arquetípicos de esta corriente, mientras que Madrazo no realizaría hasta mucho más tarde, concretamente hasta las décadas de 1840 y 1850 , los retratos plenamente románticos que harían de él uno de los principales representantes del movimiento en nuestro país. Ese desfase que puede apreciarse entre ambas disciplinas es la causa de que El Artista sea mucho más avanzado en lo literario, con artículos dedicados a la defensa del Romanticismo, relatos como los que acabamos de mencionar de Ochoa y poemas de autores tan representativos como José Zorrilla (1817-1893), José de Espronceda (1808-1842) y Jacinto de Salas y Quiroga (1813-1849). En cambio, en lo concerniente a las Bellas Artes siempre tuvo un posicionamiento más conservador, evidente, por ejemplo, en los artículos plenamente admirativos que dedicó a arquitectos de la órbita clasicista como Juan de Herrera (1530-1597) o Juan de Villanueva (1739-1811), en vez de llevar a cabo una defensa a ultranza de las construcciones góticas, apreciable en otras publicaciones de la misma época como No Me Olvides. 
Este doble rasero por parte de El Artista al referirse a lo romántico, que en definitiva corrobora asimismo la tardía generalización del Romanticismo en España, prueba también que resulta mucho más sencillo considerarse adalid de un determinado movimiento en todas sus manifestaciones que predicar con el ejemplo. Es algo que no se encuentra presente en el caso de The Germ. En relación con esto, de nuevo hemos de tener en cuenta que las fechas no son las mismas, pues este rotativo vio la luz quince años después, como tampoco la manera en que la corriente romántica arraigó en Inglaterra. Esto se había producido mucho antes que en nuestro país, comenzando en la década de 1790 tanto en lo literario, con exponentes como William Wordsworth (1770-1850) y Samuel Coleridge (1772-1834), como en lo artístico, con John Constable (1776-1837) y Joseph Mallord William Turner (1775-1851). En consecuencia, lo que los prerrafaelitas trataron de llevar a cabo desde 1848 no fue sino un epígono del Romanticismo próximo al Realismo, de ahí que muchos presupuestos de los que se hicieron eco en The Germ (como la revalorización de lo medieval, el interés por la naturaleza, etc.) ya hubieran sido asimilados por la anterior generación romántica.

Por otro lado, la dualidad arte-literatura también permite constatar diferencias en cuanto a lo que más interesaba a los equipos directivos. The Germ apostó decididamente por lo literario, alcanzando un porcentaje del 86\% del total de artículos publicados: solo siete estaban dedicados a las Bellas Artes, mientras que los otros cuarenta y tres tenían relación con la literatura. No obstante, conviene recordar que para los prerrafaelitas las dos cosas estaban necesariamente interconectadas y por ello no es extraño que alguno de esos artículos literarios haga también referencia a lo pictórico. Por el contrario, en El Artista, aunque lo que más interesaba era asimismo lo literario, los porcentajes no muestran un contraste tan acusado: de los cuatrocientos ocho artículos publicados, doscientos veintidós versaban sobre la literatura (un 54\%) y ciento dieciséis sobre las Bellas Artes (un $28,4 \%$ ). El resto de textos (setenta, un 17,2\% del total) se referían a otras cuestiones que igualmente interesaban a El Artista, principalmente la música, la historia y los artículos de costumbres, lo que denota un carácter más pluridisciplinar que, pese a estar menos acentuado que en las publicaciones divulgativas de las siguientes dos décadas, lo diferencia decididamente del carácter más especializado de The Germ.
En lo concerniente al formato de ambas publicaciones, podemos apreciar de nuevo similitudes y diferencias. Es interesante constatar, por ejemplo, que los distintos tamaños, además de condicionar la disposición del texto (a dos columnas en El Artista con artículos breves completando los huecos dejados por los largos, a una columna en The Germ con artículos de aproximadamente la misma extensión), demuestran cuál era la mentalidad de sus equipos directivos. En el caso de la revista española lo que estos pretendían era ajustarse a los parámetros de los rotativos franceses más exitosos; en el de la inglesa, emular la altura intelectual de un recopilatorio de ensayos.

Algo en lo que sí coinciden es en el hecho de considerarse unas revistas de lujo, circunstancia derivada de que sus directores fueran artistas interesados en dotarlas de un preciosismo extremo. De ahí su elevado precio y la enorme importancia concedida a los grabados, realizados en ambos casos mediante la técnica litográfica a fin de dotarlos de un acabado lo más pictórico posible. No podía ser de otro modo dado que, como hemos explicado, sus artífices fueron en muchos casos pintores de reconocido prestigio, motivo por el cual los grabados acabaron convirtiéndose en uno de los principales reclamos de los suscriptores de las revistas, llegando incluso a desentenderse del resto del contenido.

El análisis de estas similitudes y diferencias permite alcanzar unas conclusiones muy claras con respecto a ambas revistas. Si bien es cierto que El Artista y The Germ se asemejan absolutamente en cuanto a sus orígenes, su evolución acabó avanzando por derroteros distintos convirtiéndolas por tanto en ejemplos de dos maneras antitéticas de entender la prensa artística en el siglo XIX. The Germ constituye un proyecto mucho más coherente, en el que sus miembros se mantuvieron fieles durante los cinco meses de vida de la revista a los presupuestos románticos que pretendían difundir, entre otras cosas, porque para entonces estaban totalmente afianzados en Gran Bretaña. El Artista, por el contrario, en su afán por atraer a un número cada vez más amplio de lectores, sin tratar de convertirse pese a ello en una revista de corte divulgativo, fue renunciando de manera progresiva a esos ideales que en su momento había abrazado con entusiasmo, de ahí que en lo concerniente a las Bellas Artes no podamos hablar de una publicación tan posicionada de parte del Romanticismo. 
Creemos que esta relativa falta de compromiso de El Artista fue lo que le permitió sobrevivir unos meses más que The Germ, aunque realmente ambas revistas constituyen un buen ejemplo de las aventuras periodísticas emprendidas en el siglo XIX por jóvenes entusiastas que acabaron fracasando debido a problemas económicos. Pero también en esto podemos apreciar una mayor coherencia por parte del equipo de de The Germ, que cuando dejó de existir este rotativo no se embarcó en ninguna otra publicación, en comparación con el de El Artista, que, como hemos visto, volvió a ponerse once años más tarde al frente de una nueva publicación titulada El Renacimiento cuyo posicionamiento era mucho más conservador que su predecesora, prueba de hasta qué punto habían cambiado por entonces los intereses de Madrazo y Ochoa debido a su mayor estabilidad profesional.
Con independencia del grado de compromiso que pueda apreciarse en ambas, es incuestionable que desempeñaron un papel muy importante que sí las coloca a la misma altura: el de pioneras en el ámbito de las revistas artísticas de corte romántico, fugaces y desapercibidas en el momento de su publicación pero valoradas en su justa medida con el transcurso del tiempo. Por otro lado, las reediciones, ediciones críticas y facsímiles que han visto la luz a lo largo del siglo XX dan buena cuenta de cómo los estudios modernos acerca de la historiografía artística decimonónica han sabido apreciar la originalidad de esta clase de publicaciones y su importancia como difusoras de las Bellas Artes, en el momento en que estas abandonaron el ámbito académico para pasar a ser un tema de dominio público.

\section{Bibliografía}

ALONSO CABEZAS, María Victoria (2014), «La imagen del artista en la prensa periódica del siglo XIX: El Artista y Semanario Pintoresco Español», Boletín de arte, n. ${ }^{\circ}$ 35, Universidad de Málaga, pp. 101-116.

ALONSO SEOANE, María José (2013), «La participación del Conde de Campo Alange en "El Artista” en los tres primeros meses de 1835", Anales de literatura española, n. ${ }^{\circ} 25$, pp. 11-43.

ASHCROFT NOBLE, James (1882), "A Pre-Raphaelite Magazine», Fraser's Magazine, n. ${ }^{\circ}$ 25, pp. 568-580.

AYALA ARACIL, María de los Ángeles (2002), "La defensa de lo romántico en la revista literaria El Artista», en AA. W., Romanticismo 8. Actas del VIII Congreso Los románticos teorizan sobre sí mismos (Saluzzo, 21-23 de marzo de 2002), II Capitello del Sole, Bolonia, pp. 35-42.

BLANCO MARTíN, Miguel Ángel (1988), «Opinión pública y libertad de prensa (1808-1868)», en AA. W., La prensa española durante el siglo XIX. I Jornadas de especialistas en prensa regional y local, Instituto de Estudios Almerienses, Granada, pp. 27-118.

BULLEN, J. Barrie (2009), «Mid-Nineteenth-Century British Primitivism and the Continent of Europe», en ORESTANO, Francesca y FRIGERIO, Francesca, Strange Sisters: Literature and Aesthetics in the Nineteenth Century, Peter Lang, Oxford, pp. 59-76.

CALVO SERRALLER, Francisco y GONZÁLEZ GARCíA, Ángel (1981), «Estudio preliminar», en AA. W., El Artista (edición facsímil), I, Turner, Madrid, pp. IX-XXIX.

COOK, Edward Tyas y WEDDERBURN, Alexander (coords.) (1904), The Works of John Ruskin, XII, Library Edition, Londres.

GALLEGO GALLEGO, Antonio (1990), Historia del grabado en España, Cátedra, Madrid.

HARTZENBUSCH, Juan Eugenio (1894), Apuntes para un catálogo de periódicos madrileños desde el año 1561 al 1870, Establecimiento tipográfico Sucesores de Ribadeneyra, Madrid.

HOLMES, John (2015), «Pre-Raphaelitism, Science, and the Arts in The Germ», Victorian Literature and Culture, 43, pp. 689-703.

LAFUENTE FERRARI, Enrique (1987), Breve historia de la pintura española, II, Akal, Madrid.

LLORENS, Vicente (1989), El Romanticismo español, Castalia, Madrid.

PALMEGIANO, Eugenia M. (2013), Perceptions of the Press in Nineteenth-Century British Periodicals: A Bibliography, Anthem Press, Londres. RADFORD, Ernest (1898), «The Life and Death of The Germ», Idler, 13, pp. 227-233.

READ, Benedict, BARNES, Joanna y CHRISTIAN, John (coords.) (1991), Pre-Raphaelite Sculpture: Nature and Imagination in British Sculpture, 1848-1914, Henry Moore Foundation y Lund Humphries, Londres. 
RIEDE, David (2007), «The Pre-Raphaelite School», en CRONIN, Richard, CHAPMAN, Alison y HARRISON, Antony H. (coords.), A Companion to Victorian Poetry, Blackwell Publishers, Oxford, pp. 305-320.

RIEGO, Bernardo (2001), La construcción social de la realidad a través de la fotografía y el grabado informativo en la España del siglo XIX, Servicio de Publicaciones de la Universidad de Cantabria, Santander.

RODRÍGUEZ GUTIÉRREZ, Borja (2004), «El Artista arrepentido: El Renacimiento de 1847», Voz y letra: Revista de literatura, XV, n. ${ }^{\circ} 1$, pp. $77-98$.

- (2011), «La voluntad iconográfica y aristocrática de El Artista», Revista de literatura, LXXIII, 146, pp. 449-488.

ROSE, Andrea (1992), The Germ: the Literary Magazine of the Pre-Raphaelites, Ashmolean Museum, Oxford.

ROSSETTI, William Michael y FREDEMAN, William Evan (1975), The P. R. B. Journal: William Michael Rossetti's Diary of the Pre-Raphaelite Brotherhood, 1849-1853, together with other Pre-Raphaelite Documents, Clarendon Press, Oxford.

SIMÓN DÍAZ, José (1947), «El Artista de París y El Artista de Madrid», Revista Bibliográfica y Documental, n. ${ }^{\circ}$, pp. $261-267$.

STAUFFER, Andrew M. (2012), «The Germ», en PRETTEJOHN, Elizabeth (coord.), The Cambridge Companion to the Pre-Raphaelites, Cambridge University Press, Cambridge, pp. 76-85.

SURIANO, Gregory R. (2005), The British Pre-Raphaelite Illustrators, Oak Knoll Press, New Castle.

TOBIN, Thomas J. (2005), «Introduction», Worldwide Pre-Raphaelitism: Critical Theory, Popular Culture, Audiovisual Media, State University of New York Press, Nueva York, 2005, pp. 1-18.

TORRES NEBRERA, Gregorio (2012), «Ochoa y Montel, Eugenio», en AA. W., Diccionario Biográfico Español, XXXVIII, Real Academia de la Historia, Madrid, pp. 241-243. 